\title{
أثثر العولمة على نمو الناتج المحلى الإجمالي بالدول العربية الإفريقية
}

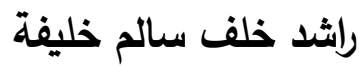

طالب دراسات عليا (دكتوراه)، بمعهد البحوث والدراسات الإفريقية ودول حوض النيل جامعة

$$
\text { أسوان. }
$$

$$
\text { جلال عبدالفتاح الصغير }
$$

أستاذ الاقتصاد الزراعي، كلية الزراعة، جامعة أسيوط.

$$
\text { عبدالوكيل تحم أبو طالب }
$$

وكيل معهد بحوث الاقتصاد الزراعي، مركز البحوث الزراعية. 
المقدمة: لقد سجل النظام العالمي في العقدين الماضيين تحولات ملحوظة ، تجدها عوامل مثل التكامل الاقتصادي العالمي المتسارع وإعادة التثكيل التاريخي للقوى الاقتصادية وزيادة التعاون بين بلدان الجنوب. وقد كانت هذه التحولات مدفوعة بالاتجاهات الضخمة مثل التحول العميق في الخصائص الديموغرافية. التحضر السريع الأهمية المتزايدة لحركات المجتمع المدني العالمية، ظهور طبقة وسطى أكبر في البلدان الجنوبية مما أدى إلى الدد من الفقر التاريخي، وقبول حدود أنماط الاستهلاك الحالية التي تؤثر على قدرة تجديد الكوكب. ومع ذلك، كان على هذه الاتجاهات الإيجابية أن تتافس مع عدد من حالات العجز المقلقة، بما في ذلك تعميق عدم المساواة، واستمرار الفقر، ونمو العاطلين عن العمل، والقيود الخختلفة على التتقل البشري. أصبحت العولمة ظاهرة كاسحة تعيد تثكيل مجتمعات العالم من خلال الآليات الاجتماعية والاقتصادية والسياسية والثقافية بهدف دمج جميع اقتصاديات العالم في السوق الرأسمالية العالمية. في الوقت نغسه شها العالم سلسلة من التغييرات المهمة ، كان أهها أزمة دولة الرفاهية. انهيار الاتحاد السوفيتي والكتلة الاشتراكية ، فثل استراتيجيات وبرامج التتمية التي تقودها الدولة في البلدان النامية في الجنوب مما أدى إلى أزمات ديونها. في حين تم توضيح سبب الأول من حيث أزمات تراكم رأس المال، كان السبب الثاني والثالث هو غياب الديمقراطية. ولتعزيز اندماج البلدان النامية في السوق العالمية ، كان عليها أن تتنىى إصلاحات اقتصادية وسياسية ، حددها إلى حد كبير صندوق النقد الدولي والبنك الدولي. تضمنت أجندة الإصلاح الرئيسية تدابير اقتصادية مثل التحرير المالي. خصخصة القطاع العام ، وخفض الإنفاق العام على الخدات الاجتماعية ، والتدابير 
السياسية مثل "دمقرطة" النظم السياسية من خلال اعتماد السياسة التعددية وحرية أكبر للمجتمع المدني.

كظاهرة عبر وطنية، تتجاوز العولمة الحدود الوطنية. وبالتالي، فإن دور الدولة، كونها

حاجزا أمام حرية الحركة للشركات عبر الوطنية، يجب أن يتراجع. بالإضافة إلى ذلك، يفترض هدف دمج جميع اقتصاديات العالم في سوق عالمي يسيطر عليه رأس المال الدولي دورًا ضئيلًا للدولة. في هذه الأثناء، تُند مسؤولية الإصلاحات الاقتصادية والسياسية ذات الصلة بالعولمة بأثرها السلبي على غالبية السكان إلى الدولة ، التي تميل إلى اللجوء إلى الإجراءات الاستبدادية القسرية للحفاظ على قوتها وسيطرتها.

في هذا السياق، بينما تحركت الدول العربية الإفريقية التي تبنت سياسات الإصلاح بشكل أسرع نسبيًا في تتفيذ التحرير الاقتصادي، فقد كانت بطيئة في إدخال الليبرالية السياسية، وأبدت مقاومة قوية للديمقراطية الحقيقية. تم تبني الديمقراطية الليبرالية التشيلية رسمياً، وإن كان ذلك بآليات للحفاظ على سيطرة الدولة بالتحالف مع النخبة الاقتصادية الجديدة. وفيما يتعلق بمنظمات المجتمع المدني، ومعظمها من المنظمات غير الحكومية، فإن وضعها القانوني كفل لها سيطرة وثيقة من قبل الحكومات. بالإضافة إلى ذلك ، لا تثجع الحكومات إلا على تشكيل منظمات غير حكومية للرعاية الترويجية وتقديم الخدمات والتتمية الزائفة تؤدي دورًا وظيفيًا في الحد من التأثير السلبي للحرب ، ولو جزئًا. سياسات العولمة ، تهئة التوتر الاجتماعي ، وبالتالي ، تساعد على الحفاظ على الوضع الراهن.

مثكلة البحث: لقد أصبح من مستلزمات العولمة وانتقال وتبادل رؤوس الأموال وانفتاح الأسواق، السعى من أجل تغيير مفهوم الدولة وفتح المجال أمام القطاع الخاص للقيام بتحريك عجلة الاقتصاد 
الوطنى بعيداً عن بيروقراطية مؤسسات الدولة حسب مؤيدى ظاهرة العولمة. وفى بعض الحالات ينغى على الدولة أيضاً التخخل لضبط الأسواق، ففى ظل العولمة الاقتصادية لا تستطيع الدول النامية النهوض بمنتجاتها المحلية الناشئة إلا في حال وضع قيود وضوابط تحد من التأثيرات السلبية لانفتاح الأسواق غير المبرمج. مما سبق تبرز مشكلة الدراسة وهى ما هى الأساليب التى تتعها الدول العربية الإفريقية للإدارة الاقتصادية فى ظل العولمة وتغيراتها، وكيف أثرت أساليب الادارة الاقتصادية المختلفة على اقتصاديات تلك الدول فى ظل العولمة. هدف البحث: إن الطبيعة الخاصة للمنطقة العربية اليوم مستمدة إلى حد كبير من ثروتها النفطية. وقد كان النفط فاعلاً في النصف الثاني من القرن العشرين في تثكيل كل المجتمعات العربية. وكان لها تأثير قوي على تطوير كل من الدول النفطية غير النفطية من خلال هجرة اليد العاملة، والتي تسارعت خلال السبعينيات مع الطفرة في أسعار النفط. ومع ذلك، هناك عوامل أخرى أثرت على التكوينات الاجتماعية والاقتصادية للدول العربية، مثل علاقتها بالدول الرأسمالية الغربية، بالإضافة إلى التغيرات الاجتماعية والاقتصادية والسياسية الجذرية الناتجة عن الثورات التي حدثت في بعض الدول العربية في الخمسينيات والستينات. وتعتمد استجابة مختلف الدول العربية للعولمة إلى حد كبير على: الظروف الاجتماعية والاقتصادية والسياسية الأولية لكل دولة، ومستويات تطورهم المختلفة ودرجة اندماجهم الفعلي في الاقتصاد العالمي. ومع ذلك، فإن الثكل الأخير للعولمة له سماته ومظاهره وآلياته للتكامل العالمي. بالنظر إلى الطبيعة الجلية لعملية العولمة، من الضروري تسليط الضوء على العمليات الأخيرة التي بدأت من خلالها المنطقة العربية مع البلدان النامية الأخرى اندماجها الرسمي في عملية العولمة الأخيرة. لذلك يهدف البحث بثكل اساسى الى دراسة الآثار 
المختلفة للعولمة على الناتج المحلى الاجمالى لاهم الدول العربية الافريقية من خلال قياس النمو الاقتصادى وتكوين راس المال واجمالى الناتج المحلى فى المدى الزمنى القصير واثر العولمة عليهح. الطريقة البحثية ومصادر البيانات: يعتمد نموذج النمو المستخدم على نظرية كلاسيكية جديدة بسيطة لنموذج Harrod Domar الذي يربط الزيادات في الناتج (الناتج المحلي الإجمالي) بالزيادات في مدخلات رأس المال (CAP)، والعمل (LAB)، والانفتاح التجاري (TOP) والمتغيرات الأخرى مثل التغيرات التكنولوجية والمؤسسية. باستخدام المعادلة الخطية، تأخذ دالة الإنتاج (OTH)

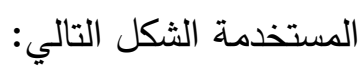
InGDPt $=\mathrm{a} 0+\mathrm{a} 1 \mathrm{InCAPt}+\mathrm{a} 2 \operatorname{InLABt}+\mathrm{a} 3 \operatorname{InTOPt}+\mathrm{a} 4 \mathrm{InOTHt}+\mathrm{et}$ a1 > 0; a2 > 0; a3 > 0; a4 < or > 0

يتم قياس المخرجات عن طريق الناتج المحلي الإجمالي، بينما يتم عن رأس المال عن طريق إجمالى التكوين الرأسمالى. بالنظر إلى الرأي القائل بأن العمالة وفيرة في الدول العربية وغيرها من البلدان النامية ، فإننا نستبعد سلسلة العمل من النموذج بينما يتم تضمين سلسلة الديون كعوامل أخرى تؤثر على النمو. بعد الدليل التجريبي على محددات النمو الاقتصادي (GDP) في البلدان النامية ، يكون نموذج النمو المقدر للتحليل على الثكل التالي: InGDPt $=$ b0 + b1 In INVt + b2InTOPt + b3FOPt + b4InDEBTt + et $\mathrm{b} 1>0 ; \mathrm{b} 2>0 ; \mathrm{b} 3>0 \mathrm{~b} 4>0$ حيث يعبر INV عن الاستثمار الذى تم قياسه عن طريق إجمالى التكوين الرأسمالى ، يتم استخدام مقياسين للانفتاح لتحديد ددى التكامل. المقياس الأول هو التكامل التجاري الذي يتم قياسه بمجموع الصادرات والواردات (TOP) والثاني هو التكامل المالي الذي يتم قياسه بمجموع تدفقات رأس المال 
الأجنبي وتدفقاته الخارجية (FOP)، و DEBT هو سلسلة الديون والمتغيرات الأخرى كما تم تعريفها مسبقًا. قد ينتج عن تقدير المعادلة (r) أعلاه بطريقة المربعات الصغرى العادية انحدارًا زائفًا إذا لم تكن المتغيرات ثابتة. من أجل التغلب على هذه المشكلة، تخضع جميع المتغيرات لاختبار جذر الوحدة من أجل تحديد خصائص السلاسل الزمنية. نحن نستخدم اختبار جذر الوحدة المعدل - ديكي فولر (ADF) على جميع المتغيرات ذات الاهتمام. كما ندرس العلاقات طويلة المدى بين الناتج والاستثمار الأجنبي المباشر وجميع محداتها. يتم ذلك أيضًا عن طريق اختبار ما إذا كانت بقايا المعادلة المقدرة (r) ثابتة لتأكيد ما إذا كانت السلسلة بالفعل مع النمو الاقتصادي. مرة أخرى ، يستخدم اختبار ADF في إجراء اختبار التكامل المشترك. ثم نستخدم تقنية نموذج تصحيح الخطأ لاشتقاق النماذج التي يتم استخدامها بعد ذلك في مزيد من التحليل. يغطي التحليل الفترة VECM

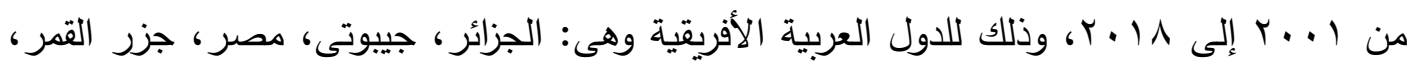
ليبيا، المغرب، موريتانيا، الصومال، السودان، تونس. بينما تم استبعاد كل من جيبوتى، ليبيا، والصومال، وذلك لعدم توفر البيانات الخاصة بمتغيرات النموذج المستخدم. بلغ عدد المشاهدات المستخدمة فى الدراسة 190 مشاهدة لكل متغير من متغيرات الدراسة. الجدول التالى يوضح متغيرات الدراسة ومصادر الحصول عليها: جدول ( إ) تعريف المتغيرات

\begin{tabular}{|c|c|c|}
\hline مصدر البيانات & التعريف & المتغير \\
\hline WDI & إجمالى الناتج المحلى الحقيقى & النمو الإقتصادى (GDP) \\
\hline WDI & إجمالى التكوين الرأسمالى & الإستثمار (INV) \\
\hline WDI & تم قياسه بمجموع الصادرات والواردات & التكامل التجاري (TOP) \\
\hline FSEDD & تم قياسه بمجموع تدفقات رأس المال الأجنبي الداخله وتدفقاته الخارجة & التكامل المالى (FOP) \\
\hline
\end{tabular}




\begin{tabular}{|c|c|c|}
\hline FSEDD & إجمالى الديون الخارجية على كل دولة & 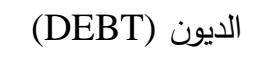 \\
\hline WDI & النسبة المئوية للتضخم السنوى بأسعار المستهلك & التضخم (INFL) \\
\hline WDI & GDP / اجمالى قيمة الصادرات والواردات & الانفتاح التجارى (T) \\
\hline
\end{tabular}

ملاحظات: WDI مؤشرات التتمية الدولية، منشوره عن طريق البنك الدولى.

FSEDD

http://data.worldbank.org/indicator/NY.GDP.DEFL.KD.ZG

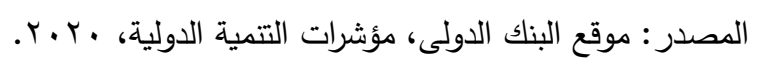

وحسب الفروض السابقة فى متغيرات النموذج القياسى تصبح معادلة الانحدار كما يلى:

GDP $=$ F $($ INVT, FOP, TOP, DEPT)

استخدمت الدراسة اللوغاريتم الطبيعى لمتغيرات الدراسة، حتى تأخذ العلاقة بين المتغير التابع

والمتغيرات المستقلة تأخذ الصور الخطية. كم يفرض اللوغاريتم الطبيعى تأثير نسبة ثابتة لمعامل المتغيرات المستقلة على المتغير التابع. كما استخدمت العديد من الدراسات السابقة اللوغاريتم الطبيعى لتقليل والحد من التحيز الذى يمكن أن ينشأ من استخدام وحدات مختلفة بين المتغيرات المستقلة والمتغير التابع.

لتحليل العلاقة السببية الديناميكية بين القطاع المصرفى المتمثله فى دوره التمويلى والتنمية

الاقتصادية، تم استخدام إطار الانحدار الذاتى الموجه .Vector Autoregressive Framework VAR) وحتى يتم تحديد نموذج VAR بطريقة صحيحة، تم اتباع الإجراءات القياسية لتحليل (Augmented Dickey-Fuller. السلاسل الزمنية. أولاً، تم تطبيق اختبار ديكى فولر المعدل (ADF) لإختبار جذور الوحدة للمتغيرات، وذلك لمعرفة خصائص استقرار السلسلة الزمنية ورتبة التكامل لكل متغير • ولتوضيح أكثر، يقال للمتغير أنه متكامل من الدرجه (d) وتكتب (d)، Id) عندما يتطلب أخذ الفروق للمتغير عدد مرات (d) حتي تصل السلسلة الزمنية للمتغير إلى حالة السكون وتصبح سلسلة ساكنة، لذلك يقال أن المتغير غير ساكن في حالة أنه متكامل من الدرجة ا أو أعلى منها. أصبح تصنيف المتغيرات إلى ساكنه وأخرى غير ساكنة من الأمور الهامة والحاسمة منذ أن 
اعتمدت الإجراءات الإحصائية القياسية في التحليل على السلاسل الساكنة فقط. وفى حال كانت السلسلة غير ساكنة فيرمز لها بالرمز (0)، أي أن السلسلة ليس بها جذر وحدة قبل اجراء عملية أخذ الفروق، وفى حال تحول السلسلة إلى الإستقرار بعد أخذ الفرق الأول يرمز للسلسلة فى هذه الحالة بالرمز (1)، وذلك يعنى أن السلسلة متكاملة من الدرجة الأولى (Wooldridge, 2012). (Johansen, فى الخطوة الثانية من التحليل تم استخدام اختبار التكامل المشترك لجوهانسن (1988. يقدم هذا الاختبار معلومات عما اذا كانت متغيرات الدراسة الخاصة بدور التطاع الدصرفى فى التتمية الاقتصادية مرتبطة ببعضها البعض فى الددى الطويل أم لا. فى نفس الوقت تمدنا اختبارات جذور الوحدة والتكامل المشترك بمعلومات مهمة تكون بمثابة المرشد لتحديد نموذج الاندار الذاتى الموجه الأنسب لطبيعة المتغيرات ومدى ارتباطها ببعضها البعض، ليحدد العلاقة السببية والديناميكية بين متغيرات الدراسة. ثالثاً: في حال كانت المتغيرات غير ساكنة تظهر المشكلة الإحصائية الرئيسية وهى وجود انحدار زائف لا يعبر عن طبيعة العلاقة بين المتغيرات، لذلك تستخدم طريقة الفروق لتحويل السلاسل الغير ساكنة إلى سلاسل ساكنة. وفى حال حدوث ما سبق لجميع متغيرات الدراسة، أى أنها كانت غير ساكنة في صورتها الأصلية ثم تحولت إلى السكون بعد أخذ الفرق الأول، هنا يجب استخدام الانحدار الذاتى الموجه (VAR) فى صورة نموذج معامل تصحيح الخطأ Vector Error) Correction Model. VECM) $\Delta \mathrm{y}_{\mathrm{t}}=\beta_{0}+\sum_{i=1}^{n} \beta i \Delta Y_{\mathrm{t}-1}+\sum_{i=0}^{n} \delta i \Delta \mathrm{x}_{\mathrm{t}-1}+\varphi \mathrm{z}_{\mathrm{t}-1}+\mu_{\mathrm{t}}$ Zحدد تصحيح الخطأ (ETC) وتعبر عن البواقى للمربعات الصغرى العادية من انحدار التكامل المشترك فى الددى الطويل، كما هو معبر عنه بالمعادلة التالية: 
$\mathrm{y}_{\mathrm{t}}=\beta_{0}+\beta_{1} \mathrm{x}_{\mathrm{t}}+\varepsilon_{\mathrm{t}}$

وتعرف كالآتى:

$\mathrm{Z}_{\mathrm{t}-1}=\mathrm{ECT}_{\mathrm{t}-1}=\mathrm{y}_{\mathrm{t}-1}-\beta_{0}+\beta_{1} \mathrm{x}_{\mathrm{t}-1}$

ب, بعبر عن سرعة التعديل لأنها تقيس السرعة التى يعود بها المتغير Y بعد التغيرات التى تحدث فى المتغير X.

وقد تم الاعتماد على مؤشرات التنمية الدولية، والهيكل المالى وقاعدة بيانات التنمية

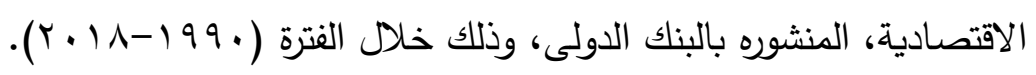

العولمة والدول العربية الإفريقية: على الرغم من الاختلافات في التكوين الاجتماعي والاقتصادي والسياسي للمجتمعات العربية، وعلى الرغم من المحاولات المختلفة لاستراتيجيات تعزيز التتمية على مدى العقود الخمسة الماضية، لا تزال التتمية هي التحدي الرئيسي للدول العربية. أصبحت العولمة في العقود الأخيرة عاملاً بارزًا، وسياقًا تأثرت فيه جميع القضايا الأخرى، وخاصة التتمية والديمقراطية، بثكل كبير · وبما أن المفاهيم يتم تحديدها اجتماعياً وتاريخياً، فهي معيارية ويتغير معناها ومضمونها وفقًا للسياق التاريخي والاجتماعي. وبالتالي، من الضروري تحديد المفاهيم الرئيسية، أي العولمة والتتمية والديمقراطية فيما يتعلق بالظروف الاجتماعية التاريخية لمجتمعات أفريقيا العربية في هذا المنعطف التاريخي. العولمة على الرغم من تأثيرها الثامل فهى لا تزال واحدة من القضايا الأكثر إثارة للجدل، سواء على المستوى الفكري والسياسي. على الرغم من وجود عدة تعريفات للعولمة، إلا أن لها تعريفين لهما نهجان مختلفان: التعريف الوظيفي والبنائي، ويمكن تقسيم كلا التعريفين أيضًا على طول خط الاختلاف بين خطاب العولمة والإيديولوجية، من ناحية، والعولمة كعملية تاريخية من 
ناحية أخرى. علاوة على ذلك، يميل كل تعريف إلى إنتاج إستراتيجية متسقة مع افتراضاته المنطقية والمنهجية.

التعريف الوظيفي وصفي وعرضـي. وتتناول العولمة من خلال وصف مظاهرها وإنجازاتها مثل الإنجازات التكنولوجية والعلمية الكبيرة، وتقدم المعلومات والاتصالات، وقوة وديناميكية الشركات عبر الوطنية، والسوق العالميـة المفتوحـة المهيمنة التي تضـن حريـة حركة رأس المـال والمنتجات، والخدمات والعمالة. الخطاب الثقافي والأيديولوجي لهذا التعريف هو نشر العولمة باعتبارها انتصار الحضارة الغربية التي تمثل نهاية التاريخ (عبد الفضيل، . . . ب). وفي هذا السياق، يتم تقديم العولمة على أنها الظاهرة التي لا مفر منها والتي تحول العالم إلى قريـة عالمية. لذلك، يجب على أولئك الذين يرغبون في البقاء في هذا العالم إعادة تأهيل أنفهه ومجتمعاتهم وإعادة هيكلتها وتعديلها حتى يصبحوا لائقين بما فيه الكفاية ليتم قبولهم وإدماجهم في النموذج القائم، أي السوق العالمية كنتيجة للحضـارة الغربية. إن دعاة هذا النهج لا يهتمون بالمصالح المتاقضـة القائمسة بين أجزاء مختلفة من العالم الناشئة عن عدم المساواة الهيكلية، ولا مع تهميش محتمل أو حتى تأثير العولمة على بعض المجتمعات، ولا التتاقضـات المتأصلة في عملية العولمـة نفسها. في هذا السياق، المبدأ الاجتماعي

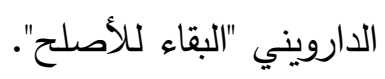

ومع ذلك، فإن المتبعون لهذا النهج الأكثر إنسانية لا تزال تؤمن بالجودة "التي لا مفر منها" المتأصلة في عملية العولمة، ولكنها لا تدرك فقط تأثيرها الإيجابي على الجنس البشري، ولكنها تدرك أيضًا تأثيره السلبي المحتمل. وفي هذا الصدد، تحدد إستراتيجية العولمة ذات الصلة بالتعريف الوظيفي الهدف النهائي باعتباره تكامل جميع اقتصاديات العالم في السوق العالمية. ولتحقيق هذا الهدف، ينبغي إعادة هيكلة الاقتصاديات النامية وتعديلها، حتى تكون مؤهلة للتكامل العالمي، باتباع 
وصفة عالمية صاغها خارج مجتمعاتها المدراء العالميون لعمليات العولمة. ومع ذلك، فإن الوعي المتزايد بالتأثرات السلبية للعولمة التي أبرزها تقرير اليونيسف بعنوان "العولمة بوجه إنساني"، لفت الانتباه إلى التشويش المحتمل لآليات العولمة إذا لم يتم اتخاذ إجراءات متضافرة للحد من هذه الآثار السلبية. وفي هذا السياق، أضافت الرؤية الوظيفية للعولمة إلى سياساتها الداعمة لاستراتيجيتها بهدف تسهيل وتقليل آلام العولمة قدر الإمكان.

التعريف الهيكلي للعولمة هو تحليلي وديناميكي وموجه تاريخياً. بينما يتعامل مع مظاهر العولمـة، فإنـه يحلل آلياتها والأسـاس المنطقي لتطورها، من منظور تاريخي. في هذا السياق، تقدم العولمة كعملية تاريخية جدلية، ومرحلة متقدمة من تاريخ البشرية المتغير باستمرار، من حيث المعرفة العلمية التراكمية والتكنولوجيا، وبالتالي فهي ليست نهاية التاريخ. كما أنها مرحلة متقدمة في تطور الرأسمالية، تقوم على المستويات التفاضلية وغير المتكافئسة لتتميـة المجتمعـات العالميـة المختلفـة، وبالتالي تخلق تقسيمًا عالميًا جديدًا للعمل يتميز بعلاقات قوة غير متكافئة اقتصاديًا وسياسيًا على نطـاق عـالمي. يؤكد أنصـار هذا النهج على أن العولمـة، بحكم طبيعتها مستقطبة، أي أن منطق التوسع الرأسمالي العالمي ينتج تفاوتًا متزايدًا بين أعضـاء النظام. وبالتالي لا يمكن أن يكون هنالك آليات للحاق من داخل النظام. إن اللحاق بالركب من التطور المتأخر يأتي من سياسات فك الارتباط مما يعني الخضوع لعلاقات أي مجتمع مع السوق العالمية إلى المتطلبات الأساسية للتمية الداخلية لذلك المجتمع (Amin. 1999). إن فك الارتباط بهذا المعنى هو المفهوم المعاكس لـ "التكيف" مع الاتجاهات العالمية لأن مثل هذا التعديل من جانب واحد، بحكم الضرورة، يؤدي إلى زيادة هامش / تهميش الأعضاء الضعفاء في النظام. يعني فك الارتباط أيضًا أن تصبح وكيلًا نشطًا في تثكيل العولمة عملية جعلها 
تتكيف مع متطلبات التطور الثخصي. يجب أن يكون واضعي السياسات الوطنيين قادين على تحديد السياسات الاقتصادية على أساس الأولويات الوطنية الفعلية والاحتياجات المستقبلية. وبالتالي ينغي أن تنثأ السياسات عن المصالح الوطنية وتعبر عنها. وبهذا المعنى ينغغي أن تكون العولمة التي ستفيد البلدان النامية تشاركيه أصيلة بآليات دافعة نحو المساواة الاجتماعية والبشرية. تفترض العولمة التكامل العالمي من خلال حرية حركة أسواق السلع والخدمات ورأس المال والعمل. ومع ذلك، فإن أسواق السلع والخدمات ورأس المال فقط هي التي تمكنت من الثروع في التكامل العالمي، في حين لا يزال سوق العمل مجزأ. هذه الظاهرة تزيد من عدم المساواة العالمية من خلال الاستغلال التفاضلي للعمال على أساس تجزئة سوق العمل. ويزداد هذا الأمر سوءًا بسبب سياسات الهجرة التقييدية التي تتنباها دول الثمال المتقدمة. لذلك، يجب دراسة الآثار الاستقطابية للأسواق العالمية بعناية أكبر • بما أن رأس المال، وخاصة "رأس المال المالي"، أصبح متحركًا إلى ما لا نهاية، وكان العمل متحركًا جزئًا فقط، فلا ينبغي النظر إلى عملية العولمة على أنها مرادفة لسوق العمل العالمية على المستوى العالمي. إن الطبيعة الخاصة للمنطقة العربية اليوم مستمدة إلى حد كبير من ثروتها النفطية. كان النفط فاعلاً في النصف الثاني من القرن العشرين في تثكيل كل المجتمعات العربية. كان لها تأثير قوي على تطوير كل من النفط والدول غير النفطية من خلال هجرة اليد العاملة، والتي تسارعت خلال السبعينيات مع الطفرة في أسعار النفط. ومع ذلك، هناك عوامل أخرى أثرت على التكوينات الاجتماعية والاقتصادية للدول العربية، مثل علاقتها بالدول الرأسمالية الغربية، بالإضافة إلى التغيرات الاجتماعية والاقتصادية والسياسية الجذرية الناتجة عن الثورات التي حدثت في بعض الدول العربية في الخمسينيات والستينات. تعتمد استجابة مختلف الدول العربية للعولمة إلى حد ما على: 
الظروف الاجتماعية والاقتصادية والسياسية الأولية لكل دولة. مستويات تطورهم المختلفة ودرجة اندماجهم الفعلي في الاقتصاد العالمي.

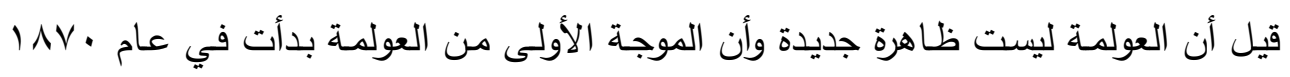
من خلال الأنثطة التجارية والاستثمارات الخارجية. بعد ذلك، جاءت الموجة الثانية من العولمة التي بدأت في السبعينيات من خلال عمليات الشركات عبر الوطنية في إطار عملية "تدويل الإنتاج" (عبد الفضيل. . . . ץ). كان لكل موجة من العولمة مركز انتشر فيه نفوذها في جميع أنحاء العالم. يقترح أن المنطقة العربية كانت مركزًا مشعًا للعولمة بهذا المعنى أكثر من مرة عبر التاريخ. ومع ذلك، منذ ظهور الحضـارة الغربيـة الحديثة، أصبحت المنطقة العربيـة متلقيـة سلبية لتأثير العولمـة. في عـام 1991 مر قرنان منذ أول اتصـال بين المنطقة العربية و"الغرب الحديث" من خلال حملة نـابليون على مصـر • بعد بضـع سنوات، افتتح محمد علي جولـة جديدة مـن التفاعل بين الاقتصـاد المصـري والاقتصادي العالمي من خلال تصدير القطن إلى أوروبا واستيراد الإنتاج والمعرفة وفنون الحرب من أوروبـا. شـهـ القرن التاسـع عشر والعقود الأولى مـن القرن العشرين سلسـلة مـن اندماجات الـول العربيـة، الواحدة تلو الأخرى، في الاقتصـاد العالمي من خـلال الاحتلال البريطاني أو الفرنسي أو الهيمنة الأمريكية. وقد أثرت هذه العمليات بشدة على المنطقة العربية اقتصاديا واجتماعيا وسياسيا وثقافيا (Amin. 1999).

ومع ذلك، فإن الثكل الأخير للعولمة له سماته ومظاهره وآلياته للتكامل العالمي. بالنظر إلى الطبيعة الجدلية لعملية العولمة، من الضروري تسليط الضوء على العمليات الأخيرة التي بدأت من خلالها المنطقة العربية مع البلدان النامية الأخرى اندماجها الرسمي في عملية العولمة الأخيرة. 
ويعكس الوضع في المنطقة العربية بقدر أكبر من الوضوح الدور الضروري للدولة، وإن كان

ذلك على أساس الديمقراطية كثرط مسبق، حيث أن بعض الدول العربية لا تزال في مرحلة بناء الدولة بدون مؤسسات اقتصادية أو سياسية أو اجتماعية قوية لتحل محلها الدولة في إدارة الثؤون العامة. ومع ذلك، ينبغي أن يكون واضحًا أن أي تطور ناجح يتطلب مشاركة شعبية حقيقية من خلا آليات ديمقراطية مؤسسية وتثاركية.

فيما يتعلق بالدور الاقتصادي للدولة، حتى في الدول المنتجة للنفط على الرغم من هيمنة

اقتصاديات السوق الحرة فإنها تتنيى استراتيجيه تتمية الرفاهية التي تقودها الدولة. إن تركيز الثروة في الدولـة يفوق بكثير الدخل الخـاص والدـدخرات. وهذا يضـع الدولـة المركزيـة في وضـع فريد لتخصيص الموارد لأنشطة التمية الثاملة. يستثر عدد من هذه البلدان بشكل مكثف في المشاريع الصناعية والخدمية الناجحة نسبيًا. تستفيد الاستثمارات الأخرى من إعانات الدولة في سياق خطط التتمية لأهداف اقتصادية واجتماعية محددة جيداً (عبد الغني. 999 ).

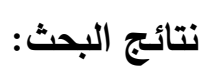
-نتائج اختبار ديكى فولر المعل لجذور الوحدة (اختبار السكون): كما تم الإثارة اليه سابقاً، يعتبر فهم طبيعة البيانات من الأمور الأساسية عند اجراء تحليل الانحدار. لذا قامت الدراسة بتطبيق (اختبار السكون) على جميع متغيرات الدراسة باستخدام برنامج EViews 10 قبل الانتقال إلى المرحلة التالية في التحليل القياسى وهى اختبار جوهانسن للتكامل المشترك. كل المتغيرات بالدراسة كانت ذات جذور وحدة. لذلك استخدمت الدراسة الفرق الأول لتحويل المتغيرات الغير ساكنة إلى

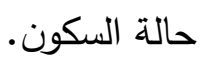


يوضح جدول (r) نتائج اختبار السكون، حيث تبين أن جميع المتغيرات غير ساكنه فى المستوى (0)، بينما أثارت نتائج اختبار ديكى فولر المعدل سكون جميع المتغيرات بعد أخذ الفرق الأول. كما هو موضح فإن قيم P بالجدول (Y) تثير إلى رفض الفرض الصفرى عند مستوى معنوية 1\%، 0 \% لجميع المتغيرات بعد أخذ الفرق الأول.

جدول (r) نتائج اختبار ديكى فولر المعدل

\begin{tabular}{|c|c|c|}
\hline 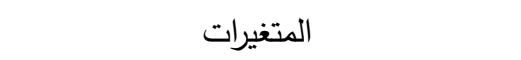 & ADF & P-VALUE \\
\hline Ln GDP (النمو الاقتصادى) I(0) & 0.452 &..$r r 1$ \\
\hline Ln Y(النمو الاقتصادى) I(1) & $\varepsilon .741$ & $0.021 *$ \\
\hline Ln K (التكوين الرأسمالى) I (0) & -1.653 & 0.0523 \\
\hline Ln K (التكوين الرأسمالى) I (1) & -5.321 & $0.000 * *$ \\
\hline Ln n (نمو السكان) I(0) & -0.432 & 0.251 \\
\hline Ln n (نمو السكان) I(1) & -3.052 & $0.000 * *$ \\
\hline Ln LIQ (الاعتمادات المالية) at I(0) & -4.321 & 0.321 \\
\hline Ln LIQ (الاعتمادات المالية) at I(1) & -7.215 & $0.000 * *$ \\
\hline Ln DC (الإقراض المحلى) I(0) & -1.242 & 0.231 \\
\hline Ln DC (الإقراض المحلى) I(1) & -4.324 & $0.000 * *$ \\
\hline Ln PC (التمان القطاع الخاص) I(0) & -1.362 & 0.342 \\
\hline Ln PC (ائتمان القطاع الخاص) I(1) & -5.234 & $0.001 * *$ \\
\hline Ln INFL (التضخم) I(0) & -2.821 & 0.423 \\
\hline Ln INFL(التضخم) I(1) & -6.325 & $0.000 * *$ \\
\hline Ln T (الانفتاح التجارى) I(0) & 0.452 &.$M r I$ \\
\hline I(1) Ln T & $\{.74$ & $0.021 *$ \\
\hline
\end{tabular}

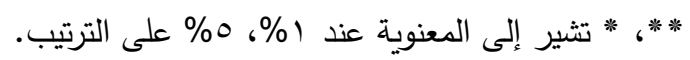

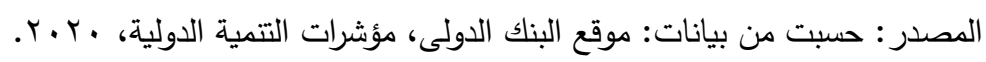
http://data.worldbank.org/indicator/NY.GDP.DEFL.KD.ZG -نتائج اختبار التكامل المشترك: بعد إجراء اختبار السكون، وظفت الدراسة اختبار جوهانسن للتكامل المشترك باستخدام برنامج EViews 10 وذلك لفحص العلاقة فى المدى الطويل بين النمو الاقتصادى متمثلاً فى إجمالى الناتج المحلى ومتغيرات الدراسة الاقتصادية الأخرى. يمكن القول أن 
هناك تكامل مشترك اذا اجتمعتا سلسلتان زمنيتان أو أكثر من متغيرات الدراسة فى الصورة الخطية، ويشترط لإجراء اختبار جوهانسن للتكامل أن تكون جميع المتغيرات بالدراسة غير ساكنة عند المستوى، ولكنها تصبح ساكنة بعد أخذ الفرق الأول لتلك المتغيرات.

يتكون اختبار جوهانسن من: maximum rank و eigenvalue و trace statistic. يحدد

عدد المعاملات أو المعادلات المتكاملة معاً عند تقدير انحداراً يحتوى على اثنان maximum rank أو أكثر من المتغيرات التوضيحية، عند maximum rank للصفر لا يكون هناك أى تكامل. أما maximum فتحدد المعادلات المتكاملة الموجودة عند كل مستوى من مستويات trace statistic trace statistic توجد معادلة التكامل عند النقطة التى يكون عندها القيمة الحرجة rank من

Maximum rank نتائج اختبار جوهانسن للتكامل المشترك: توضح نتائج جدول (ץ) أنه عند (race statistics أق)، تكون القيمة الحرجة من ه\%. لذلك يتم رفض الفرض الصفرى الذى يشير إلى عدم وجود تكامل مشترك بين متغيرات الدراسة، مما يؤكد وجود تكامل مشترك بين متغيرات الدراسة بكينيا، وكذلك وجود علاقة بين المتغيرات فى المدى الطويل. جدول (ץ) نتائج اختبار جوهانسن للتكامل المشترك.

\begin{tabular}{|c|c|c|c|c|c|c|c|}
\hline $\begin{array}{c}\text { Maximum } \\
\text { rank }\end{array}$ & $\begin{array}{c}\text { Eigenvalu } \\
\mathrm{e}\end{array}$ & $\begin{array}{c}\text { Trace } \\
\text { Statistic }\end{array}$ & $\begin{array}{c}5 \% \\
\text { Critical } \\
\text { Value }\end{array}$ & $\begin{array}{c}\text { P- } \\
\text { VALU } \\
\text { E }\end{array}$ & $\begin{array}{c}\text { Max- } \\
\text { Eigen } \\
\text { Statistic }\end{array}$ & $\begin{array}{c}5 \% \\
\text { Critical } \\
\text { Value }\end{array}$ & $\begin{array}{c}\text { P- } \\
\text { VALU } \\
\text { E }\end{array}$ \\
\hline None* & 0.225990 & $\begin{array}{c}71.1379 \\
6\end{array}$ & $\begin{array}{c}69.8188 \\
9\end{array}$ & 0.0391 & $\begin{array}{c}38.4255 \\
3\end{array}$ & $\begin{array}{c}33.8768 \\
7\end{array}$ & 0.0134 \\
\hline At most $1 *$ & 0.104879 & $\begin{array}{c}32.7124 \\
2\end{array}$ & $\begin{array}{c}47.8561 \\
3\end{array}$ & 0.5727 & $\begin{array}{c}16.6194 \\
7\end{array}$ & $\begin{array}{c}27.5843 \\
4\end{array}$ & 0.6127 \\
\hline At most $2 *$ & 0.049828 & $\begin{array}{c}16.0929 \\
5\end{array}$ & $\begin{array}{c}29.7970 \\
7\end{array}$ & 0.7057 & $\begin{array}{c}7.66688 \\
9\end{array}$ & $\begin{array}{c}21.1316 \\
2\end{array}$ & 0.9229 \\
\hline At most $3 *$ & 0.039438 & $\begin{array}{c}8.42606 \\
4\end{array}$ & $\begin{array}{c}15.4947 \\
1\end{array}$ & 0.4210 & $\begin{array}{c}6.03556 \\
5\end{array}$ & $\begin{array}{c}14.2646 \\
0\end{array}$ & 0.6086 \\
\hline At most 4 & 0.015810 & 2.39049 & 3.84146 & 0.1221 & 2.39049 & 3.84146 & 0.1221 \\
\hline
\end{tabular}


\begin{tabular}{|l|l|l|l|l|l|l|l|}
\hline & & 8 & 6 & & 8 & 6 & \\
\hline
\end{tabular}

* تشير إلى النقطة التى يوجد عندها التكامل المشترك

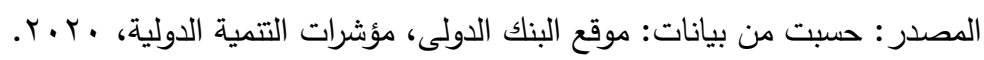
http://data.worldbank.org/indicator/NY.GDP.DEFL.KD.ZG

-نتائج نموذج معامل تصحيح الخطأ (VECM): بسبب وجود التكامل المشترك بين المتغيرات عددت الدراسة إلى التقدير بتطبيق نموذج معامل تصحيح الخطأ (VECM)، وهذا النموذج يعطى نتائج في المدى القصير وكذلك في المدى الطويل. الجزء التالى من الدراسة يوضح مدى استجابة النمو الاقتصادى للتغير فى المتغيرات المستقلة. معادلة التكامل المشترك التالية توضح تقديرات النموذج فى المدى الطويل، حيث ثبت معنوية النموذج ككل. تثير النتائج الى أن الإستثمار (INV) كان له أثراً ايجابياً على على النمو الاقتصادى فى كينيا، وتوضح النتائج أن زيادة التكوين الرأسمالى بمقدار ايزيد النمو فى إجمالى الناتج القومى (GDP) بمقدار ^r.r\% فى المدى الطويل، مع بقاء المتغيرات الأخرى على حالها. كما تشير المعادلة أيضاً الى الأثر الإيجابى للتكامل التجارى (TOP) على النمو الاقتصادى، حيث توضح النتائج أن كل زيادة ا \% فى نمو السكان يؤدى الى زيادة مقابلة فى النمو الاقتصادى بمقدار ا (Yr\%، مع بقاء المتغيرات الأخرى على حالها. وهذا ما يوضح الدور الهام الذى يلعبه كل من الاستثمار والتكامل التجارى متمثلاً في مجموع الصادرات والواردات لكل دولة، على الناتج المحلى الاجمالى للدول العربية الإفريقية.

أما بالنسبة لتأثير التتمية المالية على النمو الاقتصادى، جاءت علامات معاملاتها متباينة، حيث تثير النتائج أن التكامل المالى (FOP) والذى تم قياسه عن طريق مجموع التدفقات المالية الداخلة لكل دولة والتدفقات المالية الخارجة من كل دولة جاءت اشارتها سالبة مما يعنى أنها ذات تأثير 
سلبى على النمو الإقتصادى، مما يعنى أن زيادة مقدارها ( \% فى التكامل المالى يؤدى الى نقصان قدره 2.18\% فى النمو الاقتصادى، مع بقاء باقى المتغيرات على حالها. بينما جاءت إثارة الديون (DEPT) سالبة، مما يعكس الأثر السلبى للديون على إجمالي الناتج المحلى للدول العربية الإفريقية، والتى غالباً ما تستخدم لسداد خدمة الديون السابقة، وعند استخدام جزء منها في المشروعات القومية فإنها تستخدم بمستوى متدن من الكفاءة. حيث تثير نتائج المعادلة إلى أن كل زيادة مقدارها (\% في إجمالي الديون تؤدى إلى انخفاض في الناتج المحلي

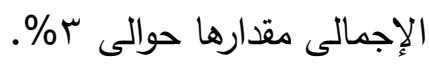
تشير الملاحظات أسفل معادلة التكامل المشترك فى (نموذج المدى الطويل) إلى كفاءة ومعنوية النموذج فى التقدير حيث بغت قيمة R المعدلة 01\%، كما تشير قيمة اختبار ديربن واتسون إلى ب 9 ـ (، مما يعنى أنه ليس هناك ارتباط ذاتى بين البواقى، حيث تأخذ قيم احصاء ديربن واتسون المدى ما بين صفر - ؛، وذلك يعنى أنه كلما اقتربنا من الصفر كلما كان هناك ارتباط ذاتى سالب، وكلما اقتربت القيمة من ؛ كان هناك ارتباط ذاتى موجب (Durbin and Watson, 1950) . وجدت قيمة معامل التصحيح U(-1) معنوية احصائياً عند مستوى معنوية 0\%، وذلك يؤكد العلاقة المعنوية بين متغيرات الدراسة في المدى الطويل، هذا بالإضافة أن قيمة معامل التصحيح بلغت .067 مما يشير إلى أن السرعة النسبية للتعديل من حالة عدم التوازن فى العام الماضى إلى حالة

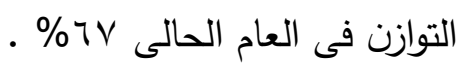
يشير جدول (ع) إلى نتائج اختبار (بريوش جودفرى) للإزتباط التسلسلى، إلى غياب هذا النوع من الترابط بين متغيرات الدراسة، مما يؤكد صحة وكفاءة النموذج فى التقدير . -معادية التكامل المشترك (نموذج المدى الطويل) 
ect $_{\mathrm{t}-1}=1.000 \mathrm{Ln} \mathrm{GDP}_{\mathrm{t}-1}+2.383 \mathrm{Ln} \mathrm{INV}_{\mathrm{t}-1}+3.706 \mathrm{Ln} \mathrm{TOP}_{\mathrm{t}-1}-2.179 \mathrm{Ln} \mathrm{FOP}_{\mathrm{t}-1}-$ 2.946 Ln DEPT $\mathrm{L}_{\mathrm{t}-1}-12.520$

$\mathrm{U}(-1)=-0.67^{*}$

Adjusted R-squared $=0.5121$

F-statistic $=6.8532 *$

Durbin-Watson stat $=1.921$

* denotes significance at $5 \%$

\begin{tabular}{|c|c|c|c|}
\hline F-statistic & 0.64594 & Prob. F(2,13) & 0.8245 \\
\hline R-squared & 3.46981 & Prob. Chi-Square(2) & 0.52314 \\
\hline
\end{tabular}

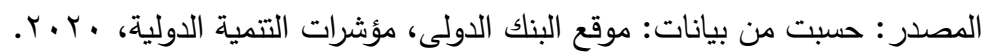
http://data.worldbank.org/indicator/NY.GDP.DEFL.KD.ZG

-العلاقة بين المتغيرات فى المدى القصير:تشير المعادلة التالية إلى العلاقة بين متغيرات الدراسة فى المدى القصير • حيث يوضح جدول (0) قيم (P) لمربع كاى باختبار إحصائيات وولد، ويتبين من الجدول أن جميع متغيرات الدراسة غير معنوية فى المدى القصير عند مستوى معنوية 0\% فيما عدا المتغيرات الخاصة الاستثمار المعبر عنه باجمالى التكوين الرأسمالى (INV). كما يوضح الجدول أن ليس لهما تأثير معنوى على النمو الاقتصادى فى المدى القصير • بينما كان إجمالى التكوين الرأسمالى (INV) وكذلك متغيرات: التكامل المالى (FOP) والديون (DEPT)، كانت جميعاً معنوية احصائياً عند مستوى معنوية أقل من ه\% ـ نستخلص من تلك النتائج أن إجمالى التكوين الراسمالى كان ذو أثز ايجابى على النمو الاقتصادى فى المدى القصير وكذلك التكامل المالى

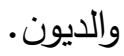

- تقدير نموذج (VECM) لمتغيرات الاراسة فى المدى القصير: $\Delta$ Ln GDP $_{\mathrm{t}}=-0.043$ ect $_{\mathrm{t}-1}+0.502 \Delta \mathrm{Ln} \mathrm{GDP}_{\mathrm{t}-1}-0.213 \Delta \mathrm{Ln} \mathrm{GDP}_{\mathrm{t}-2}+0.021 \Delta \mathrm{Ln}$ $\mathrm{INV}_{\mathrm{t}-1}-0.041 \Delta \mathrm{Ln} \mathrm{INV}_{\mathrm{t}-2}-0.241 \Delta \mathrm{Ln} \mathrm{TOP}_{\mathrm{t}-1}+0.231 \Delta \mathrm{Ln} \mathrm{TOP}_{\mathrm{t}-2}+0.231 \Delta \mathrm{Ln}$ $\mathrm{FOP}_{\mathrm{t}-1}+0.142 \Delta \mathrm{LnFOP}_{\mathrm{t}-2}-0.321 \Delta \mathrm{LnDEPT}_{\mathrm{t}-1}-0.412 \Delta \mathrm{LnDEPT}_{\mathrm{t}-2}+0.016$

جدول (0) قيم (P) لمربع كاى باختبار احصائيات وولد

\begin{tabular}{|c|c|c|c|c|c|}
\hline \multirow{2}{*}{ المتغيرات } & \multicolumn{5}{|c|}{ المتغيرات التابعة } \\
\cline { 2 - 6 } & $\begin{array}{c}\text { DLn(GDP) } \\
\text { p-value }\end{array}$ & $\begin{array}{c}\text { DLn(INV) } \\
\text { p-value }\end{array}$ & $\begin{array}{c}\text { DLn(TOP) } \\
\text { p-value }\end{array}$ & $\begin{array}{c}\text { DLn(FOP) } \\
\text { p-value }\end{array}$ & $\begin{array}{c}\text { DLn(DEPT) } \\
\text { p-value }\end{array}$ \\
\hline
\end{tabular}


راشد خلف وآخرون، أثر العولمة على نمو الناتج المحلى الإجمالي بالدول العربية الإفريقية

\begin{tabular}{|c|c|c|c|c|c|}
\hline DLn(GDP) & 0.532 & 0.352 & 0.421 & 0.321 & 0.531 \\
\hline DLn(INV) & $0.021^{*}$ & 0.241 & 0.651 & 0.452 & 0.314 \\
\hline DLn(TOP) & 0.421 & 0.433 & 0.432 & 0.314 & 0.512 \\
\hline DLn(FOP) & $0.024^{*}$ & 0.265 & 0.231 & 0.531 & 0.321 \\
\hline DLn(DEPT) & $0.015^{*}$ & $0.013^{*}$ & 0.421 & 0.231 & 0.214 \\
\hline
\end{tabular}

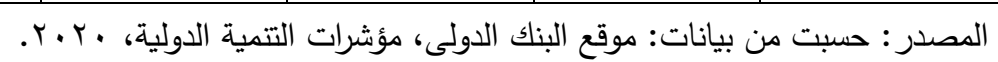
http://data.worldbank.org/indicator/NY.GDP.DEFL.KD.ZG

بحثت الدراسة تأثير العولمة على النمو الاقتصادي في الدول العربية الإفريقية بين عامي 1 . . r، ^1 ـ r . وبالنظر إلى مدى الانفتاح التجاري والتكامل المالى ، أظهرت الدراسة أن الاقتصاد العربي الإفريقى يستفيد من العولمة. كما أكدت الدراسة أن الاقتصاد لم يستفد بعد من سياسة التكامل المالي. لا تكمن المشكلة في أن الدول العربية الإفريقية مستبعدة من السوق العالمية ، ولكنها في معظد الحالات لا يتم تضمينها بالكامل فيها. الأكثر تضرراً من هذا الوضع القبيح هم الفقراء الذين لديهم أغلبية من الفلاحين والفقراء في وسط عدد قليل للغاية من الأفراد الأثرياء والفاسدين. يمارس معظم الفقراء زراعة الكفاف التي تستبعدهم من التكامل العالمي. يظل قطاع النفط في الاقتصاد هو القطاع المهيمن في المعاملات الدولية ويخلق حرمان غالبية الدول العربية الإفريقية من التمتع بفوائد الانفتاح التجاري. ويلاحظ أن معظم الدول العربية الإفريقية وضعت ونفذت سياسات اقتصادية أدت إلى فجوة أوسع بين الأغنياء والفقراء. وخلصت الدراسة إلى أنه يمكن للدول العربية الإفريقية استخدام السوق الدولية للخدمات لتحسين الحوكمة الاقتصادية وتوفير البنية التحتية اللازمة (مثل الموانئ والكهرباء) كما تشهد على قطاع الاتصالات في البلاد اليوم. وبالتالي خلصت الدراسة إلى أن المزيد من النجاحات في العقد الأول من القرن الحادي والعشرين من حيث النمو الاقتصادي من التكامل لا تتطلب فقط سياسات تجارية مفتوحة ، ولكن أيضًا مؤسسات وسياسات سليمة في مجموعة من المجالات. إذا كانت الدول العربية ، مثل أي دولة نامية أخرى ، ستقتح فقط أمام التجارة الخارجية 
والاستثمار، فإنها سوف تزدهر وتحقق النمو الاقتصادي المتسارع. سوف يشجع الانفتاح على الاستثمار الكافي من القطاع الخاص وبالتالي النمو الاقتصادي. وخلصت الدراسة إلى أن الدول العربية الإفريقية لتحقيق النمو والتتمية المتسارعة، من الضروري للغاية لدمج اقتصادها بالكامل في الاقتصادات العالمية عن طريق إزالة جميع الحواجز أمام التجارة وتحرير جميع قطاعات الاقتصاد. لقد ولدت العولمة ثروة كبيرة للدول العربية الإفريقية ويمكن استخدامها للحد بشكل كبير من الفقر وبالتالي للحد من الفقر وعدم المساواة على الصعيد العالمي. لقد ساعدت العولمة على زيادة الاستثمار وخلق الثروة في الدول العربية الإفريقية ولكن يجب تسخيرها بشكل أفضل لمساعدة الفقراء والأكثر تهميشًا على تحسين حياة مواطنيهم. ربما كان الدافع للعولمة يكمن في الديمقراطية المناسبة واقتصاديات السوق الثفافة. ويلاحظ أن الدول العربية الإفريقية بحاجة إلى دمج اقتصادها بالكامل وتحرير جميع القطاعات من أجل التمتع الكامل بفوائد العولمة. سيستغرق هذا بعض الوقت ولكن أي تراجع في الإصلاح الاقتصادي الحالي لن ينتج عنه نتائج جيدة للاقتصاد بأكمله.

الملخص أصبحت العولمة ظاهرة كاسحة تعيد تثكيل مجتمعات العالم من خلال الآليات الاجتماعية والاقتصادية والسياسية والثقافية بهدف دمج جميع اقتصاديات العالم في السوق الرأسمالية العالمية. في الوقت نفسه شهد العالم سلسلة من التغييرات المهة ، كان أههها أزمة دولة الرفاهية. انهيار الاتحاد السوفيتي والكتلة الاشتراكية ، فثل استراتيجيات وبرامج التتمية التي تقودها الدولة في البلدان النامية في الجنوب مما أدى إلى أزمات ديونها. في حين تم توضيح سبب الأول من حيث أزمات تراكم رأس المال، كان السبب الثاني والثالث هو غياب الديمقراطية. ولتعزيز اندماج البلدان النامية في السوق العالمية ، كان عليها أن تتبنى إصلاحات اقتصادية وسياسية ، حددها إلى حد كبير 
صندوق النقد الدولي والبنك الدولي. تضمنت أجندة الإصلاح الرئيسية تدابير اقتصادية مثل التحرير المالي. خصخصة القطاع العام ، وخفض الإنفاق العام على الخمات الاجتماعية ، والتدابير السياسية مثل "دمقرطة" النظم السياسية من خلال اعتماد السياسة التعددية وحرية أكبر للمجتمع

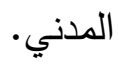

تعتد استجابة مختلف الدول العربية للعولمة إلى حد كبير على: الظروف الاجتماعية

والاقتصادية والسياسية الأولية لكل دولة. مستويات تطورهم المختلفة ودرجة اندماجهم الفعلي في ري الاقتصاد العالمي. ومع ذلك ، فإن الثكل الأخير للعولمة له سماته ومظاهره وآلياته للتكامل العالمي. بالنظر إلى الطبيعة الجدلية لعملية العولمة، من الضروري تسليط الضوء على العمليات الأخيرة التي بدأت من خلالها المنطقة العربية مع البلدان النامية الأخرى اندماجها الرسمي في عملية العولمة الأخيرة. لذلك يهدف البحث بشكل اساسى الى دراسة الاثار المختلفة للعولمة على الناتج المحلى الاجمالى لاهم الدول العربية الافريقية من خلال قياس النمو الاقتصادى وتكوين راس المال واجمالى الناتج المحلى فى المدى الزمنى القصير واثثر العولمة عليهم. Harrod Domar يعتمد نموذج النمو المستخدم على نظرية كلاسيكية جديدة بسيطة لنموذج الذي يربط الزيادات في الناتج (الناتج المحلي الإجمالي) بالزيادات في مدخلات رأس المال (CAP)، والعمل (LAB)، والانفتاح التجاري (TOP) والمتغيرات الأخرى (OTH) مثل التغيرات التكنولوجية

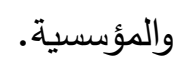
بحثت الدراسة تأثير العولمة على النمو الاقتصادي في الدول العربية الإفريقية بين عامي

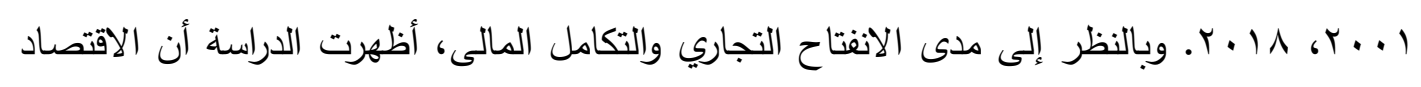
العربي الإفريقى يستغيد من العولمة. كما أكدت الدراسة أن الاقتصاد لم يستفذ بعد من سياسة التكامل 
المالي. لا تكمن المشكلة في أن الدول العربية الإفريقية مستبعدة من السوق العالمية ، ولكنها في معظم الحالات لا يتم تضمينها بالكامل فيها. الأكثر تضرراً من هذا الوضع القبيح هم الفقراء الذين لديهم أغلبية من الفلاحين والفقراء في وسط عدد قليل للغاية من الأفراد الأثرياء والفاسدين. يمارس معظم الفقراء زراعة الكفاف التي تستبعدم من التكامل العالمي. يظل قطاع النفط في الاقتصاد هو القطاع المهيمن في المعاملات الدولية ويخلق حرمان غالبية الدول العربية الإفريقية من التمتع بفوائد

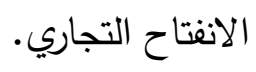

ويلاحظ أن معظم الدول العربية الإفريقية وضعت ونفذت سياسات اقتصادية أدت إلى فجوة أوسع بين الأغنياء والفقراء. وخلصت الدراسة إلى أنه يمكن للدول العربية الإفريقية استخدام السوق الدولية للخدمات لتحسين الحوكمة الاقتصادية وتوفير البنية التحتية اللازمة (مثل الموانئ والكهرباء) كما تشهد على قطاع الاتصالات في البلاد اليوم. وبالتالي خلصت الدراسة إلى أن المزيد من النجاحات في العقد الأول من القرن الحادي والعشرين من حيث النمو الاقتصادي من التكامل لا تتطلب فقط سياسات تجارية مفتوحة ، ولكن أيضًا مؤسسات وسياسات سليمة في مجموعة من المجالات. إذا كانت الدول العربية ، مثل أي دولة نامية أخرى ، ستفتح فقط أمام التجارة الخارجية والاستثمار، فإنها سوف تزدهر وتحقق النمو الاقتصادي المتسارع. سوف يثجع الانفتاح على الاستثمار الكافي من القطاع الخاص وبالتالي النمو الاقتصادي. وخلصت الدراسة إلى أن الدول العربية الإفريقية لتحقيق النمو والتتمية المتسارعة، من الضروري للغاية لدمج اقتصادها بالكامل في الاقتصادات العالمية عن طريق إزالة جميع الحواجز أمام التجارة وتحرير جميع قطاعات الاقتصاد. لقد ولدت العولمة ثروة كبيرة للدول العربية الإفريقية ويمكن استخدامها للحد بشكل كبير من الفقر وبالتالي للحد من الفقر وعدم المساواة على الصعيد العالمي. لقد ساعدت العولمة على زيادة 
الاستثمار وخلق الثروة في الدول العببية الإفريقية ولكن يجب تسخيرها بشكل أفضل لمساعدة الفقراء والأكثر تهميشًا على تحسين حياة مواطنيهم. ربما كان الدافع للعولمة يكمن في الديمقراطية المناسبة واقتصاديات السوق الثفافة. ويلاحظ أن الدول العربية الإفريقية بحاجة إلى دمج اقتصادها بالكامل وتحرير جميع القطاعات من أجل التمتع الكامل بفوائد العولمة. سيستغرق هذا بعض الوقت ولكن أي تراجع في الإصلاح الاقتصادي الحالي لن ينتج عنه نتائج جيدة للاقتصاد بأكله.

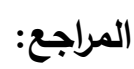
1. برهان غليون (991()، الوطن العربي أمام تحديات القرن الواحد و العشرين: تحديات كبيرة وهمم صغيرة، المستقبل العربي، بيروت، العدد 232، يوليو. ז. جال آمين (999 (1)، العولمة والتتمية العربية من حملة نابليون إلى جولة لأوروغواي، بيروت: مركز دراسات الوحدة العربية. r. جمال عبد الغني (1999)، تقييم الاستقرار الاقتصادي وسياسات التكيف الهيكلي في البلدان العربية، وقائع المؤتمر العلمي الرابع للجمعية العربية للبحوث الاقتصادية، عربي مؤسسة

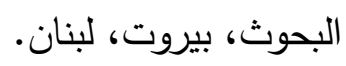
؛. السيد يس (991 ())، العرب والعولمة، مركز دراسات الوحدة العربية، بيروت. ○. محم الأطرش (ب.....)، حول تحديات العولمة الاقتصادية، مجلة المستقبل العربى، مركز دراسات الوحدة العربية، بيروت، العدد . بr.

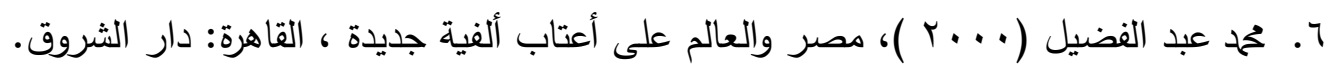

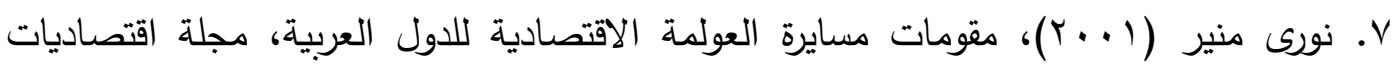

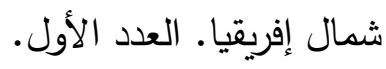


8. Amin, G., (1999), Globalization and Arab Development: From Napoleon's Campaign to the Uruguay Round 1798-1998, Beirut: Center for Arab Unity Studies.

9. Dhanapala,H. (2001). Globalization and Nation State, Global Policy Forum. Lynne Rener Publishers U.S.A.

10. Durbin, J. \& Watson, G.S. (1950), Testing for serial correlation in least squares regression I. Biometrika, 37:409-428.

11. Ishaku,B.L (2009), Globalization and the State, Unpublished Post Graduate Seminar Paper.

12. Johansen S (1988) Statistical analysis of cointegration vectors, J Econ Dyn Control 12:231-254.

13. Mkandawire, T., \& Soludo, C., (1999), Our Continent, Our Future: African Perspective On Structural Adjustment, Dakar: CODESRIA.

14. Wooldridge, J.M. (2012), Introductory Econometrics, A Modern Approach, (5th edition). South Western Cengage Learning. 


\section{Summary \\ Globalization Effect on GDP Growth in Arab African Countries}

Globalization has become a sweeping phenomenon reshaping the world's societies through social, economic, political and cultural mechanisms with the aim of integrating all the world's economies into the global capitalist market. At the same time, the world witnessed a series of important changes, the most important of which was the welfare state crisis. The collapse of the Soviet Union and the socialist bloc; the failure of state-led development strategies and programs in developing countries in the south has led to debt crises. While the first reason was explained in terms of capital accumulation crises, the second and third reason was the absence of democracy. To foster the integration of developing countries into the global market, they had to adopt economic and political reforms, largely determined by the International Monetary Fund and the World Bank.

The main reform agenda included economic measures such as financial liberalization. Privatizing the public sector, reducing public spending on social services, and political measures such as "democratizing" political systems by adopting a pluralistic policy and greater freedom for civil society.

The response of the various Arab countries to globalization depends to some extent on the primary social, economic and political conditions of each country. Their different levels of development and the degree of actual integration into the global economy. However, the last form of globalization has its features, manifestations and mechanisms for global integration. Given the dialectical nature of the process of globalization, it is necessary to shed light on the recent processes through which the Arab region with other developing countries began their formal integration into the recent process of globalization. Therefore, the research aims mainly to study the different effects of globalization on the gross domestic product of the most important Arab-African countries, by measuring economic growth, capital formation and GDP in the short term and the impact of globalization on them.

The growth model used is based on a simple new classic theory of the Harrod Domar model that links increases in output (GDP) with increases in capital inputs (CAP), labor (LAB), trade openness (TOP) and other $(\mathrm{OTH})$ variables such as technological and institutional changes.

The study examined the impact of globalization on economic growth in Arab African countries between 2001 and 2018. Given the extent of commercial openness and financial integration, the study showed that the Arab-African economy benefits from globalization. The study also confirmed that the economy has not yet benefited from the policy of financial integration. The problem is not that Arab African countries are excluded from the global market, but in most cases they are not fully included in them. Most affected by this situation Ugly are the poor who have a majority of the peasants and the poor in the midst of very few rich and corrupt individuals. Most of the poor practice subsistence agriculture that excludes them from global integration. The oil sector in the economy remains the 
dominant sector in international transactions and creates the denial of the majority of Arab African countries to enjoy the benefits of trade openness.

It is noted that most Arab African countries have developed and implemented economic policies that have led to a wider gap between the rich and the poor. The study concluded that Arab African countries can use the international market for services to improve economic governance and provide the necessary infrastructure (such as ports and electricity) as attested to by the country's telecommunications sector today. Consequently, the study concluded that more successes in the first decade of the twenty-first century in terms of economic growth than integration require not only open trade policies, but also sound institutions and policies in a range of areas.

If the Arab countries, like any other developing country, will open only to foreign trade and investment, then they will flourish and achieve accelerated economic growth. Opening up to adequate investment from the private sector and thus economic growth will be encouraged. The study concluded that Arab African countries to achieve rapid growth and development, it is absolutely necessary to fully integrate their economies into global economies by removing all barriers to trade and liberalizing all sectors of the economy. Globalization has generated a great wealth of Arab African countries and can be used to significantly reduce poverty and thus to reduce poverty and inequality at the global level. Globalization has helped increase investment and create wealth in Arab African countries, but it must be better harnessed to help the poor and the most marginalized to improve the lives of their citizens. Perhaps the drive for globalization lies in appropriate democracy and transparent market economies. It is noted that Arab African countries need to fully integrate their economy and free all sectors in order to fully enjoy the benefits of globalization. This will take some time, but any decline in the current economic reform will not produce good results for the entire economy. 